\title{
STABILITY IMPROVEMENT IN NATURAL CIRCULATION LOOP USING TESLA VALVE - AN EXPERIMENTAL INVESTIGATION
}

\author{
*U. C. ARUNACHALA ${ }^{\mathbf{1}}$, A. C. RAJAT ${ }^{2}$, DHRUV SHAH ${ }^{\mathbf{3}}$ \& UJJAWAL SUREKA ${ }^{\mathbf{4}}$ \\ ${ }^{1,3,4}$ Department of Mechanical \& Manufacturing Engineering, Manipal Institute of Technology, \\ Manipal Academy of Higher Education, Manipal, India \\ ${ }^{2}$ Department of Mechanical Engineering, National Institute of Technology Karnataka, India
}

\begin{abstract}
Natural circulation loop works on the basic principle of thermosiphon effect, which doesn't require any external force to drive the fluid in a loop. Hence they are highly applicable in power industries where there are a source and a sink. However, the flow pattern in a single-phase natural circulation loop is quite unstable especially the horizontal heater horizontal cooler configuration which results in lower loop effectiveness. A lot of research has been carried out to study these instabilities and find a way to curb them. Techniques such as tilting of loop, use of nanofluids, adding flow resistances etc. have shown promising results. But they have their own practical constraints. Hence in the present experimental study, a Tesla-type passive valve is incorporated in natural circulation loop. Due to its presence, not only stability period is reduced (at least 90 s compared to non-Tesla loop) but also unidirectional flow is achieved. The Tesla valve performed better for a broad range of heater power (90-170W) and was also able to provide noticeable resistance to the flow when the loop was inclined by $2.5^{\circ}$ in the opposite direction.

KEYWORDS: Natural Circulation Loop, Tesla Valve, Loop Stability \& Horizontal Heater Horizontal Cooler System
\end{abstract}

Received: Jul 22, 2019; Accepted: Aug 15, 2019; Published: Sep 28, 2019; Paper Id.: IJMPERDDEC20192

\section{INTRODUCTION}

The term circulation refers to a closed-circuit carrying fluid that will be under motion. Circulation or flow can be of two types viz. natural and forced. The forced type is catered by pumps, blowers etc. based on the application and fluid to be transported. The natural circulation (NC) also referred to as thermosiphon or natural convection systems which do not require any physical component to trigger its motion but needs a channel or path for it to travel. The driving force for the flow occurs due to the presence of heat source and heat sink. This is the reason why natural circulation loop (NCL) finds application in power industries, solar water heaters, transformer cooling, geothermal power extraction, cooling of internal combustion engines, gas turbine blades and nuclear reactor cores.

In heat pipe, the heating and cooling happen in the same leg/channel whereas, in NCL there are two separate legs in which fluid can take either direction once getting heated. NCL behaves differently for the various heater and cooler orientations as thoroughly researched by both numerically and experimentally. Horizontal heater horizontal cooler (HHHC) is the least stable arrangement (Figure 1) and has been researched a lot in order to improve its directional stability and reduce the time it takes to form stable flow. The fluid in the horizontal position can take either of the clockwise or anti-clockwise direction, giving rise to uncertainty and ineffectiveness. However, vertical heater vertical cooler (VHVC) is the most stable configuration as flow is readily established forming a unidirectional and stable loop. Other orientations are horizontal heater vertical cooler (HHVC) and vertical heater horizontal cooler (VHHC). 
Due to the lack of control and interplay of various forces in NCL, various kinds of instability can arise. The flow is considered stable when the parameters like velocity and temperature do not change with time. All NC systems take a certain amount of time to stabilize. The alternating effects of buoyancy and gravity due to change in density across the loop causes such oscillation. It also includes the actions of friction and velocity. The flow in HHHC type loop may take any direction, and to have a more stable and controllable system, flow need to be unidirectional.

\section{TESLA VALVE}

A Tesla valve is a valvular conduit, fixed-geometry passive check valve without moving parts. It allows fluid to flow preferentially in one direction. The device is named after Nikola Tesla, who was awarded a patent in 1920 for its invention [2]. To increase the efficiency of the Tesla valve, one has to increase the resistance in the reverse side and also have a lower pressure drop in the forward direction. In an attempt to obtain this, D-type Tesla valve is designed as shown in Figure 2. However the old version is depicted in Figure 3.

\section{LITERATURE REVIEW}

In this section, the recent literature review related to single-phase HHHC type of NCL, instabilities and restraining techniques in NCL, and Tesla valve have been discussed.

To understand the fluid behaviour, Misale et al. [4] have carried out experiments in a rectangular loop filled with water and water-glycerol mixture. It was found that the temperature variations throughout the loop followed a pattern of damped oscillations until it reached a steady state. The one-dimensional analytical procedure by Keller [5] for a loop indicated that the fluid took a particular direction and exhibited periodic motion in terms of its magnitude of velocity. These oscillations were observed to be mainly dependent on the frictional and buoyancy forces. Vijayan [6] has experimentally captured the characteristics of steady-state and stability behaviour of NCL. It was verified that the steadystate Re in uniform or non-uniform diameter NCL could be expressed as,

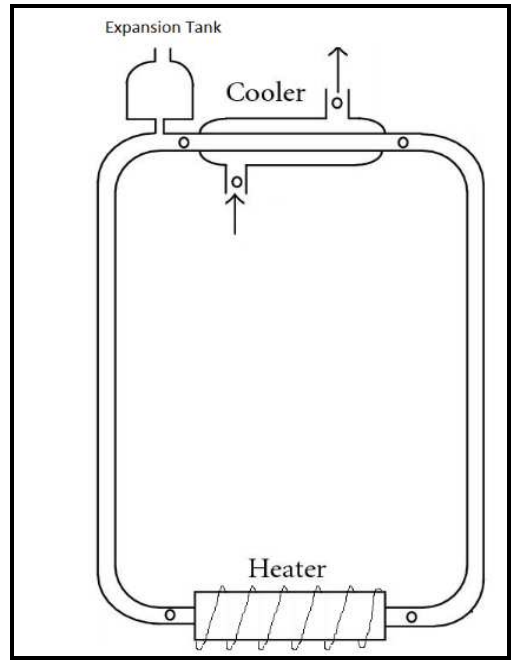

Figure 1: NCL- HHHC System [1].

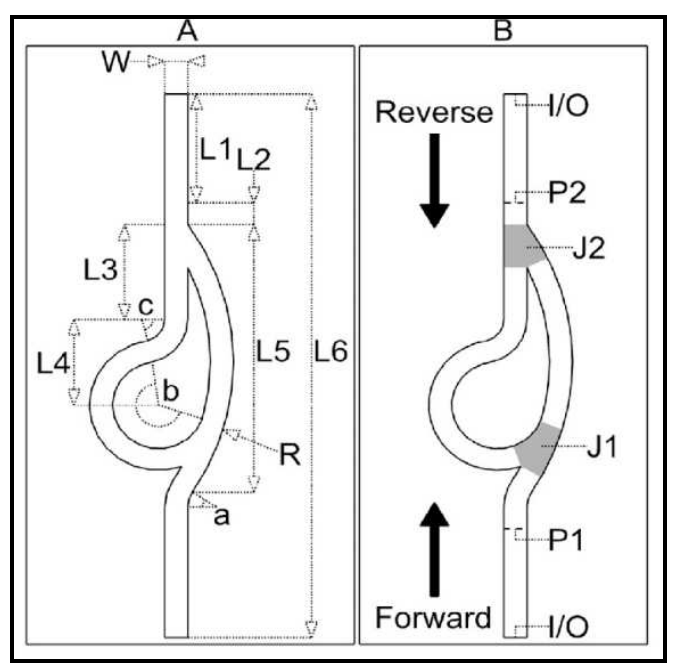

Figure 2: D-Type Tesla-Valve [3].

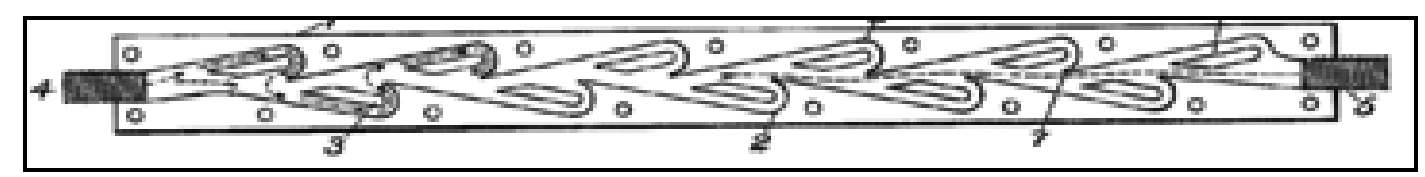

Figure 3: Cross-Section of a Tesla Valve [2]. 


$$
R e_{s s}=C\left[G r_{m} / N_{G}\right]^{\mathrm{r}}
$$

Where the constants $r$ and $C$ depend on the nature of the flow (laminar or turbulent), $N_{G}$ is the geometric ratio and $G r_{m}$ is the modified Grashoff number. Further, the stability criteria for uniform and non-uniform diameter loop was defined as a function of Stanton number, modified Grashoff number and geometric ratio. The effect of wall friction on stability of NCL in different flow regimes was numerically studied by Ambrosini et al. [7] and concluded that the friction model to be used in a loop is very much dependent on the geometry. Swapnalee and Vijayan [8] have done analytical and experimental studies with respect to four different orientations of the heater and cooler and, presented general flow equations assuming partial laminar and partial turbulent flow. The obtained equations were in good agreement with the data in earlier literary works.

Yadav et al. [9] have conducted steady-state numerical analysis in $\mathrm{CO}_{2}$ (sub-critical/super-critical) based loop with end heat exchangers. It shows that if the average temperature of the loop increases, the mass flow rate and the heat transfer rate increases until the pseudo-critical point and then decreases. Further, correlations are also proposed to predict the $\mathrm{Re}$ and $\mathrm{Nu}$ values in case of NCL with end heat exchangers. In similar study, Yadav et al. [10] have performed experimental transient analysis at sub-critical and supercritical operating conditions (50-100 bar and 323-353K) by varying loop tilt angle and external mass flow rate. It was observed that the heat transfer rate increases with increase in operating temperatures and external mass flow rate, whereas it decreases with the loop tilt angle. Additionally, it was reported that the loop is quite stable under the influence of any disturbances inflected.

Doganay and Turgut [11] studied the impact of input power, inclination and the condenser temperature on the thermal performance of a mini loop by varying the particle concentrations of $\mathrm{Al}_{2} \mathrm{O}_{3}$-DIW (De-ionised water) nanofluid. As the concentration of the nanofluids and inclination increases, the thermal effectiveness of the system improves. It was also reported that $\mathrm{Al}_{2} \mathrm{O}_{3}$-DIW nanofluid has enormous potential to overhaul the thermal management problems in large heat dissipation devices due to its better effectiveness in high-temperature conditions. Cheng et al. [12] have formulated 1-D mathematical model for loop with end heat exchangers. The formula was derived for temperature distributions along the loop. It was observed that the modified Grashoff number, heat transfer rate and Reynolds number increase with the increase in driven temperature difference. Further, Cheng et al. [13] have theoretically and experimentally studied the heat transfer and fluid flow characteristics of loop with double pipe heat exchangers. The cooling fluid temperature is fixed $\left(10^{\circ} \mathrm{C}\right)$, and the heating fluid inlet temperature was varied from $30^{\circ} \mathrm{C}$ to $60^{\circ} \mathrm{C}$. It was observed that stability reached for all the cases but the stabilizing time decreases with an increase in heating fluid inlet temperature. Upon using the $\mathrm{Nu}$ correlation for laminar flow, i.e. $\mathrm{Nu}=3.66$, significant error between the theoretical and experimental results was observed. Hence a new correlation (Equation 2) was formulated which showed much better agreement.

$$
N u=0.2702 R e^{0.4713}
$$

A lot of research highlights analyses in NCL [4-13]. However, analysis of HHHC type is critical as they showcase a twofold instability behaviour. Vijayan et al. [14] have discussed the stability of loop with different heater and condenser orientations and experimentally found VHVC and HHHC orientation as the most stable and least stable, respectively. Kudariyawar et al. [15] have done the 3-D steady and Transient state characteristics of various configurations of NCL viz. VHVC, VHHC, HHVC and HHHC, and compared with experimental data. The importance of a low Reynolds number model for CFD calculations and flow initiation transients were discussed. Additionally, the bi-directional and unidirectional pulsations in the HHHC configuration was extensively explained. 
Many researchers have discussed the instability involved in NCL and techniques to curb it. Vijayan et al. [1] had rigorous theoretical and experimental analysis to prove that stability increases as the diameter of the loop decreases. Krishnani and Basu [16] performed a computational study to see the influence of inclination angle on the stability behaviour of HHHC-type loop. It was observed that with the increase in power and sink temperature, the loop witnesses a large number of unstable oscillations and flow reversals. Providing the loop with an inclination decreases these unstable oscillations drastically. The explanation lies on the basis that upon inclination, the buoyancy force reduces by a factor of the cosine of the angle of oscillation. However, as inclination increases, the steady-state mass flow rate of the system decreases and therefore, an ideal inclination of $15^{\circ}$ was recommended for further designs of NCL systems. Instability is very prominent in NCL, especially in HHHC arrangement. Till date literature reflects various techniques to overcome this instability and uncertainty viz. varying loop diameter [1], presence of orifice [17] and use of nanofluids [18]. All mentioned instability restraining techniques have their own limitations, and hence the possibility of Tesla valve (TV) in loop has been discussed here as it is one of the most promising adaptations due to meagre maintenance requirements and uni-directional flow.

Tesla [2] invented a valvular conduit to be used for unidirectional fluid flow. He stated that mechanical valves are not reliable, and they add massively to the cost of manufacture and maintenance. The basic principle of the valvular conduit is that in the forward direction, the fluid flows without much resistance, but there is a significant amount of resistance incorporated in the fluid in the opposite direction and hence limiting the flow in the forward direction only. Forster et al. [19] discussed the design, fabrication and the testing of no moving parts valve. The CFD analysis based design was fabricated and tested positively. Truong and Nguyen [3] had objective to increase the value of diodicity of TV and hence optimized the design. It is found through flow simulation that diodicity vary non-linearly with flow rate. Gamboa et al. [20] have done TV shape optimization which resulted in significant increase in diodicity values for Reynolds number (0-2000). The experimental models were made using CNC and verified with CFD results. The numerical analysis by Zhang et al. [21] revealed that for Reynolds number up to 2000 if the hydraulic diameter is kept constant, the TV valve with a higher aspect ratio can yield better diodicity. Thompson et al. [22] have integrated Teslatype check valve to increase the thermal performance of a flat- plate oscillating heat pipe (FP-OHP). When it was introduced, the desired direction was promoted with increment in heat input. The thermal resistance was always lesser in a TV FP-OHP compared to the FP-OHP without the valve. Nobakht et al. [23] did numerical study on the diodicity mechanism of different Tesla-type microvalves and found that the major pressure loss happens during the inlet, irrespective of the direction. Hence to increase the diodicity, the flow losses in the forward direction has to be reduced. The experimental results of the microvalves were compared with numerical simulation and found good agreement. Thompson et al. [24] discussed the transitional and turbulent flow modelling of TV. The aim was to find the appropriate turbulent model which agrees the best with experimental data and to optimize the valve using the right model so as to increase the diodicity. Further, Thompson et al. [25] have done numerical analysis of multi-staged TV and found that the diodicity can increase up to 2 on having a series of TVs. This effect is prominent at higher Reynolds number. Vries et al. [26] have discussed the design and operation of TV for pulsating heat pipes. It was designed on the basis of 2-D and 3-D numerical analysis for enhancing the flow in a pulsating heat pipe. The aim was to maximize the diodicity and retain low-pressure drop in the promoted direction. In the numerical analysis, the diodicity found was higher for higher Reynolds numbers. Even Rajat [27] has mentioned the benefit of adding TV in NCL as numerical result gave unidirectional flow. 
As discussed, instability in HHHC loop is maximum and to curb it various techniques have been employed. However, such techniques have their own limitations which lead the present experimental study to incorporate TV in HHHC loop.

\section{EXPERIMENTAL ANALYSIS}

This section includes details of the expérimental setup, instruments used and the procedure of conduction.

\subsection{Experimental Setup}

The setup consists of TV connected to NCL. The TV is made by aluminium sheet with the use of EDM machine. Later, all alluminium pieces were joined by high-temperature silicon sealant and the final product is as shown in Figure 4.

The NCLs are made of stainless steel pipe of $10 \mathrm{~mm}$ inner diameter and $0.8 \mathrm{~mm}$ thickness. The loop size is $1000 \mathrm{~mm}(\mathrm{H}) \times 500 \mathrm{~mm}(\mathrm{~W})$. For condenser, $450 \mathrm{~mm}$ long PVC pipe with $25 \mathrm{~mm}$ inner diameter is used. Two such loops (with and without TV) are prepared. Each unit is having seven pre-calibrated K-type thermocouples (Heater, Heater entry/exit, Condenser entry/exit, Coolant entry/exit) and pressure tapping as shown in Figure 5. Loop fluid used is water and its expansion during heating is taken care by providing vents at the top. Differential pressure transducer (Honeywell) is connected to vertical legs for identifying flow oscillations. For heater, the tube is first wrapped with mica sheet (electrical insulation) over which Nichrome wire is wound. It is then covered with an additional layer of mica sheet. Both the loops are covered with multi-layers of glass wool for thermal insulation. Heater is connected through dimmerstat and power analyzer (Yokogawa), and mass flow meter (Rheonik) is connected to coolant pipe. Output from all instruments are given to data logger (Keysight) for real time data acquisition. The complete experiment setup with all instrumentations is shown in Figure 6.

\subsection{Experimental Procedure}

The pre-requisites to be followed before experimentation are,

- Pulsate the NCL to remove any trapped bubbles.

- $\quad$ Set the condenser mass flow rate.

- $\quad$ Pre-heat the loop to remove additional bubbles.

After all the pre-requisites are met, the following steps are followed,

- Data logger is started with scaning rate of $10 \mathrm{~s}$ to capture pressure and temperature readings (in particular).

- Set the dimmer stat to supply the known power toheater.

- Wait for steady state $(\simeq 30 \mathrm{~min})$ and repeat the steps with different power input for both the NCLs.

- After each trial, heater is switched OFF and flow in condenser is continued so that the temperature and pressure values can fall back to the initial values $(\simeq 2 \mathrm{~h})$. 


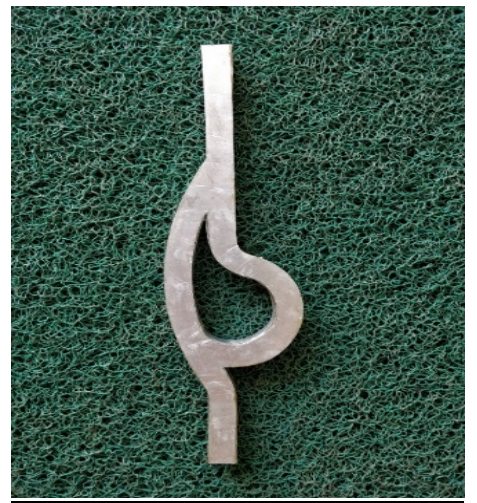

Figure 4: Fabricated TV.

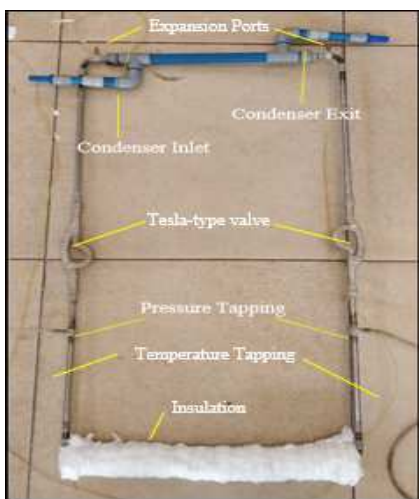

Figure 5: NCL with TV.

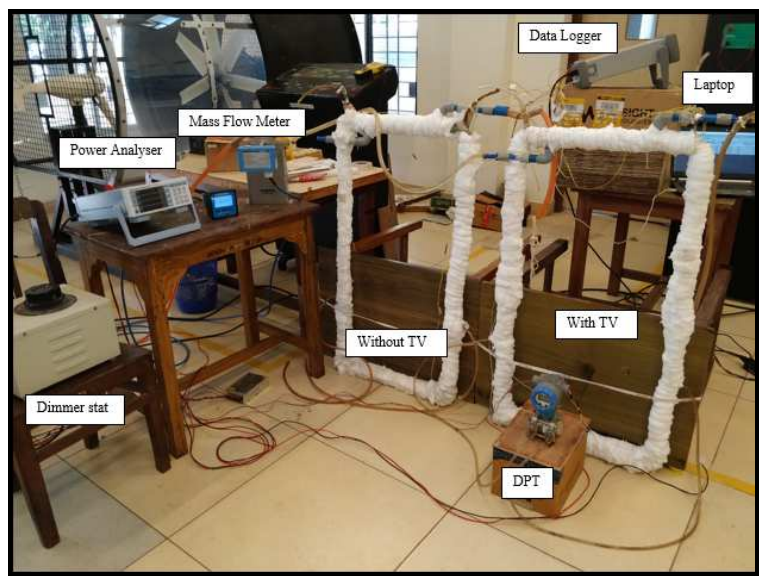

Figure 6: Experimental set up.

To test the stability of NCL, power variation made was $90 \mathrm{~W}, 110 \mathrm{~W}, 130 \mathrm{~W}, 150 \mathrm{~W}$ and $170 \mathrm{~W}$. Additionally, the loop was inclined at angles viz. $2.5^{\circ}, 5^{\circ}$ and $10^{\circ}$ to understand the working of the TV at these angles and to compare them without the TV.

\section{RESULTS AND DISCUSSIONS}

Here, the main parameters for data reduction are temperatures at different points and pressure within the loop. The stability of TV-based loop was tested in two ways, firstly by measuring the differential pressure between two legs and secondly by analyzing the variation and difference in temperature at the lower side of each leg (lower right - lower left). Pressure curve shows stability when the oscillations are reduced to minimum. A constant non-zero value of $\Delta \mathrm{T}$ signifies the formation of a loop, i.e. counterclockwise (positive) and clockwise (negative) when viewing from the front. The tests covered heater power ranging from $90 \mathrm{~W}$ to $170 \mathrm{~W}$ with a step of $20 \mathrm{~W}$.

The temperature variations give insights into the behaviour of the loop over time at different points in the loop. For a heater power of $150 \mathrm{~W}$, it can be seen from Figure 7 that the temperature profile of the loop with TV is smoother than the one without TV (Figure 8). The time taken to reach stability with TV is also less with respect to the loop without TV.

Further, in the case of TV-based loop, the $\Delta \mathrm{T}$ graph is positive, indicating fluid movement in forward direction of the Tesla. As mentioned earlier, $\Delta \mathrm{T}$ graph is smoother with TV for different heater input power. At $90 \mathrm{~W}$ power, the loop with TV has reached more stable state faster than that of the loop without TV, which shows the stabilizing effect TV has on 
NCL. Also, the high and steep peaks of the $\Delta \mathrm{T}$ and $\Delta \mathrm{P}$ of the TV during the initial stages of heating reveals that the TV is more responsive to the heating effects of the fluid as shown in Figure 9. A similar trend can be observed in the $\Delta \mathrm{P}$ values for loop without TV which is close to zero as shown in Figure 10. It means the dynamic pressure difference in the loop is negligible, and hence the flow is minimal or erratic. In the loop with TV, the pressure difference has a non-zero value, signifying the presence of noticeable amount of flow and that too in forward direction. Similarly at $170 \mathrm{~W}$ also the instability in terms of variations in temperature (Figure 11) and pressure (Figure 12) for loop without TV which takes more time to attain a constant value compared to the case of TV.

Next, $\Delta \mathrm{T}$ and $\Delta \mathrm{P}$ plots are made considering all power trials $(90 \mathrm{~W}-170 \mathrm{~W})$. They show a similar trend, having an initial peak and then after a couple of oscillations reaches more stable temperature difference. The general observation made here is the value of temperature difference which is more when the wattage is increased. In the beginning, the peak in $\Delta \mathrm{T}$ and $\Delta \mathrm{P}$ occurs due to initial heating in the horizontal heater (which can take any direction). Further, in the loop without TV it can be either of the direction and in the loop with TV, after initial heating, the flow is biased towards forward direction always and in smoother way as shown in Figure 13. The peak observed in $\Delta \mathrm{P}$ is also due to the sudden rising of the hot fluid from the lower portion to the upper portion, but as seen from Figure 14, TV-based loop performs better.

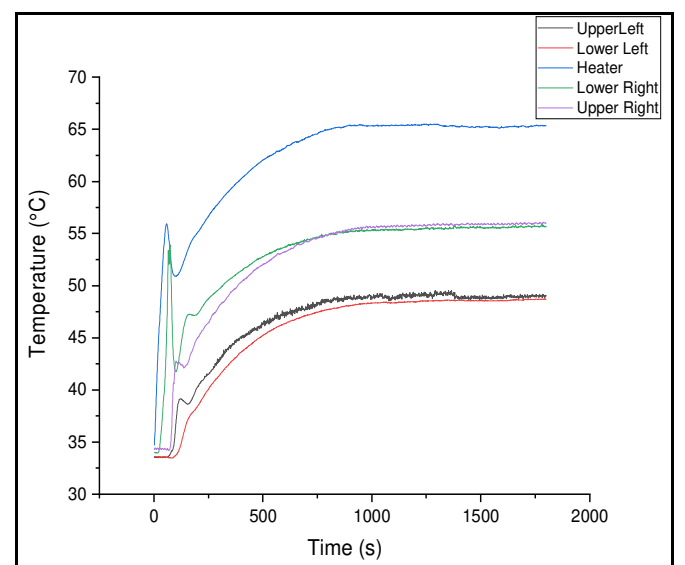

Figure 7: Temperature Profile with TV.

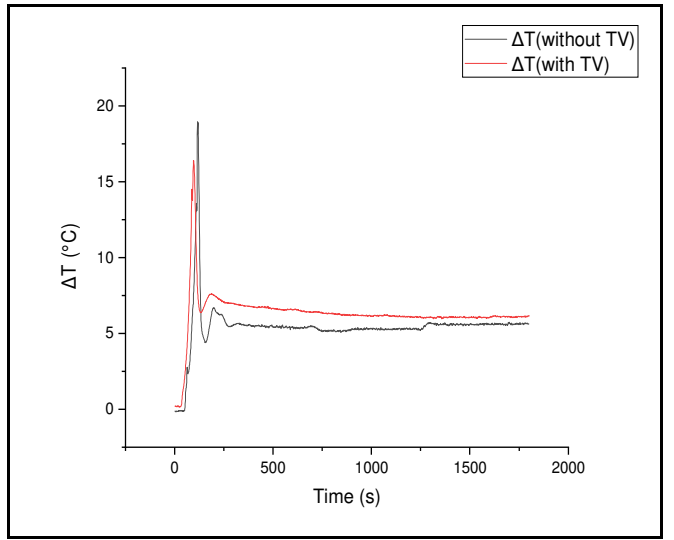

Figure 9: $\Delta \mathrm{T}$ comparison for $90 \mathrm{~W}$.

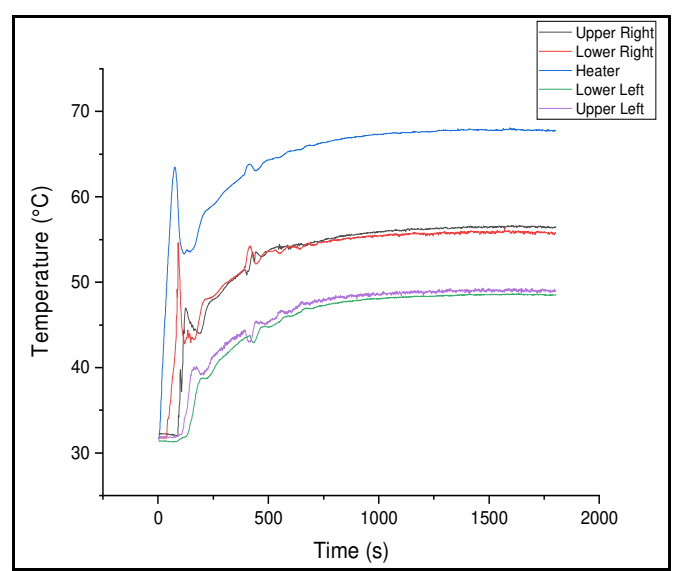

Figure 8: Temperature Profile without TV.

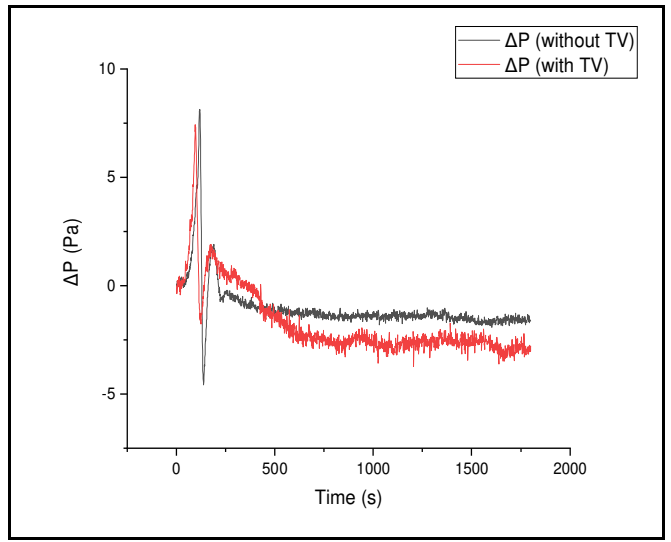

Figure 10: $\Delta \mathrm{P}$ comparison for $90 \mathrm{~W}$. 


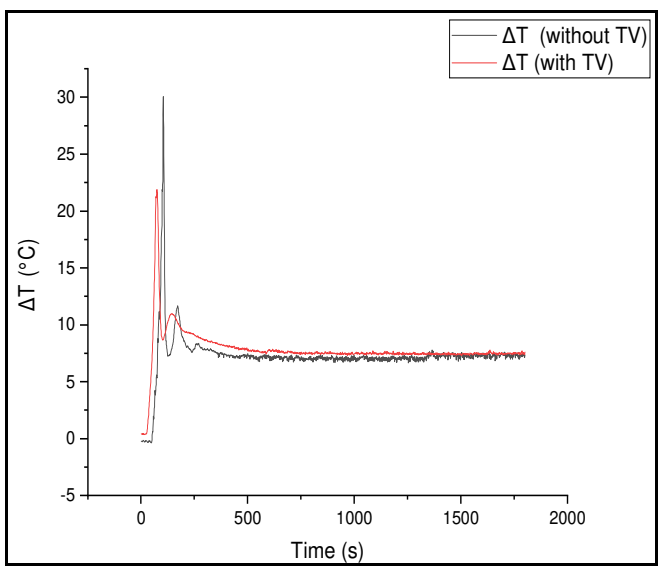

Figure 11: $\Delta \mathrm{T}$ Comparison for $170 \mathrm{~W}$.

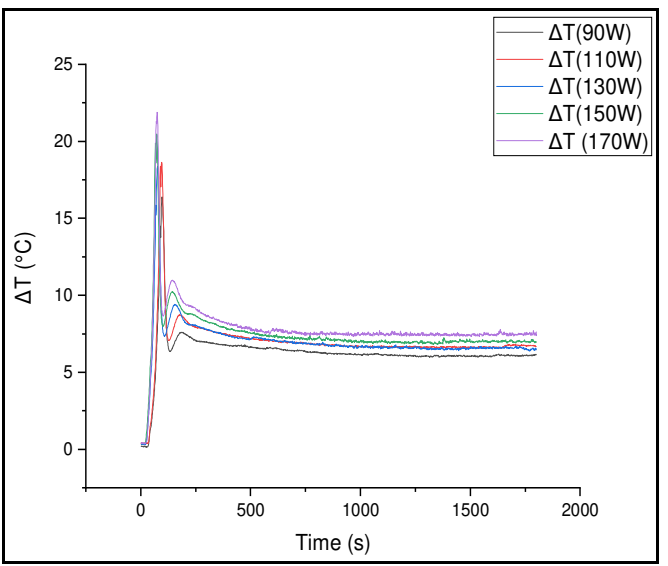

(a) Loop with Tesla valve.

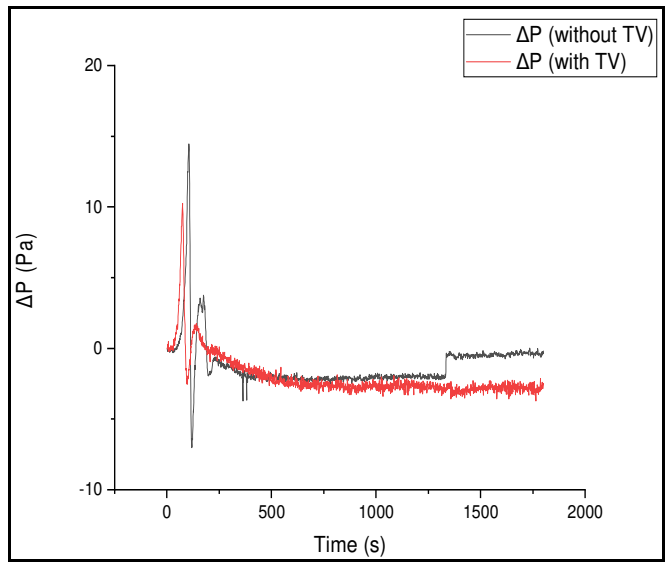

Figure 12: $\Delta \mathrm{P}$ Comparison for $170 \mathrm{~W}$.

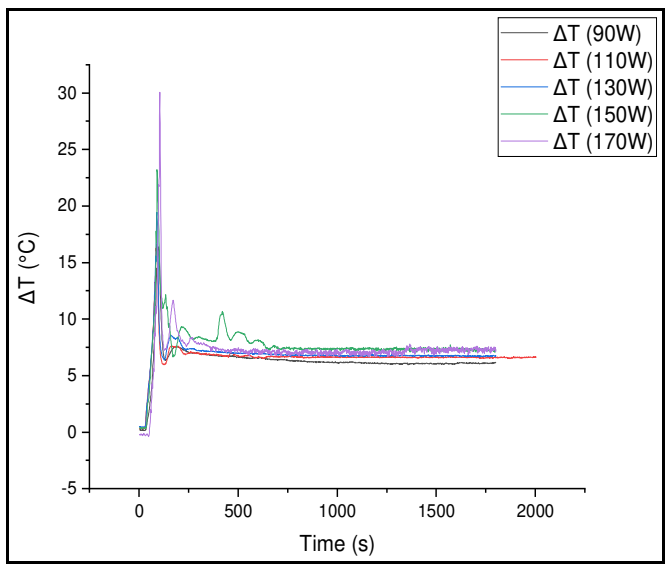

(b) Loop without Tesla valve.

Figure 13: $\Delta \mathrm{T}$ vs Time

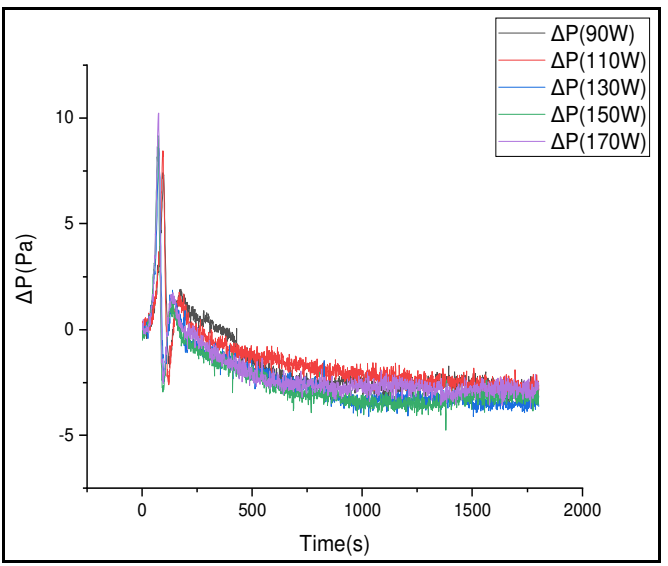

(a) Loop with Tesla valve.

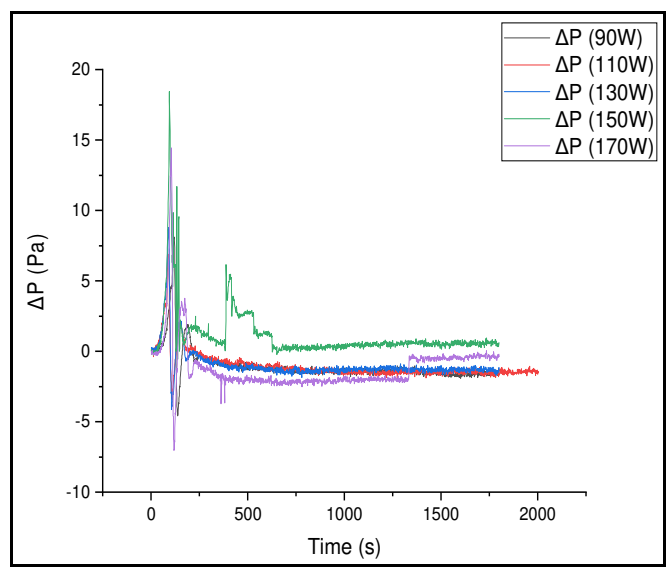

(b) Loop without Tesla valve.

Figure 14: $\Delta P$ vs Time. 

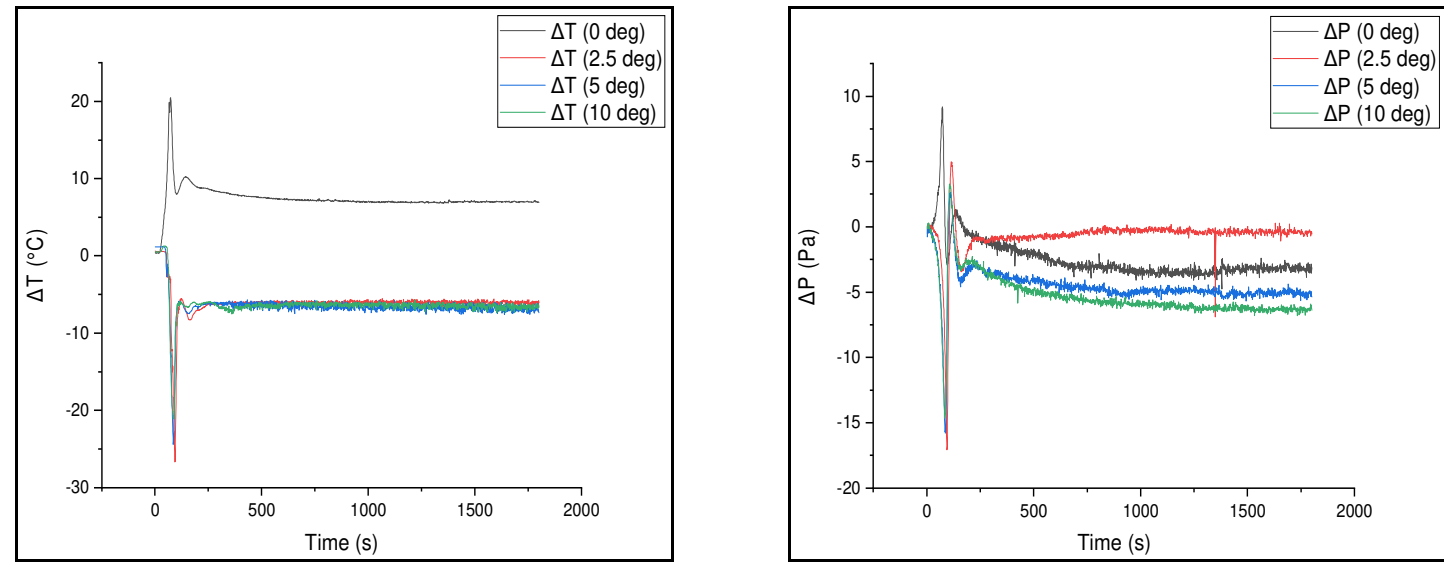

Figure 15: $\Delta \mathrm{T}$ at Different Inclinations with TV. Figure 16: $\Delta P$ at Different Inclinations without TV.

Even, $\Delta \mathrm{P}$ and $\Delta \mathrm{T}$ curves are also analyzed to understand the flow behaviour when the loop was inclined. In the loop with Tesla valve, the forward direction of the fluid is indicated by the plot with positive $\Delta \mathrm{T}$. $\Delta \mathrm{P}$ is used as an indication of the amount of flow within the loop. The effect of TV is tested at $0^{\circ}, 2,5^{\circ}$ and $5^{\circ}$ inclinations (loop was inclined so as to oppose the effect of TV and prefered the flow in the reverse direction) with $150 \mathrm{~W}$ heater power. According to the $\Delta \mathrm{T}$ graph (Figure 15$)$, the flow with $\mathrm{TV}$ at $0^{\circ}$ inclination took forward direction $(\Delta \mathrm{T}$ is positive), and the flow in the loop at other angles took reverse ( $\Delta \mathrm{T}$ is negative). This shows that TV is not able to overcome the flow bias offered due to the inclination of the loop and works better at horizontal position. From $\Delta \mathrm{P}$ graph (Figure 16), the highest flow resistance at loop angle $2.5^{\circ}$ is observed, which indicates the effect of TV. Additionally, it can't resist the flow at angle more than $2.5^{\circ}$.

\section{CONCLUSIONS}

Effect of TV in NCL was studied by using two separate loops subjected to the same test conditions. Some of the critical observations made out of the experimental investigation are summarised below.

- Directionality: For the entire range of heater power, the loop with TV showed uni-directional behaviour and favoured the flow in forward direction.

- Stability Time: From the graphs, it was observed that the time taken for the loop to reach stability was lower in the TV-based loop compared to the without TV.

- Effect of Power: The importance of a TV can be better observed on varying power input. The $\Delta \mathrm{T}$ curves for loop with TV had similar smooth profile, whereas in the loop without TV there is no observable pattern, indicating lesser control on the flow inside the loop.

- Effect of Inclination: In inclined position, the loop without TV readily took the direction favoured by the inclination. However, the loop with TV offered considerable resistance to the flow until $2.5^{\circ}$ angle of inclination.

\section{REFERENCES}

1. Vijayan, P. K., Nayak, A. K., Saha, D., \& Gartia, M. R. (2008). Effect of Loop Diameter on the Steady State and Stability Behaviour of Single-Phase and Two-Phase Natural Circulation Loops. 2008. https://doi.org/10.1155/2008/672704.

2. Nikola Tesla (1920), Valvular Conduit, United States Patent Office, US1329559. 
3. Truong, T. Q., \& Nguyen, N. T. (2003). Simulation and optimization of Tesla valves. 2003 Nanotechnology Conference and Trade Show - Nanotech 2003, 1(December).

4. Misale, M., Tagliafico, L., \& Tanda, G. (1992). Experiments in a Free Convection Rectangular Loop. Transport Phenomena in Heat and Mass Transfer, (December), 203-211. https://doi.org/10.1016/b978-0-444-89851-7.50023-6.

5. Keller, J. B. (1966). Periodic oscillations in a model of thermal convection. Journal of Fluid Mechanics, 26(3), 599-606. https://doi.org/10.1017/S0022112066001423.

6. Vijayan, P. K. (2002). Experimental observations on the general trends of the steady state and stability behaviour of singlephase natural circulation loops. Nuclear Engineering and Design, 215(1-2), 139-152. https://doi.org/10.1016/S00295493(02)00047-X.

7. Ambrosini, W., Forgione, N., Ferreri, J. C., \& Bucci, M. (2004). The effect of wall friction in single-phase natural circulation stability at the transition between laminar and turbulent flow. Annals of Nuclear Energy, 31(16), 1833-1865. https://doi.org/10.1016/j.anucene.2004.05.011.

8. Swapnalee, B. T., \& Vijayan, P. K. (2011). A generalized flow equation for single phase natural circulation loops obeying multiple friction laws. International Journal of Heat and Mass Transfer, 54(11-12), 2618-2629. https://doi.org/10.1016/j.ijheatmasstransfer.2011.01.023.

9. Yadav, A. K., Ram Gopal, M., \& Bhattacharyya, S. (2012). CFD analysis of a CO2 based natural circulation loop with end heat exchangers. Applied Thermal Engineering, 36(1), $288-295$. https://doi.org/10.1016/j.applthermaleng.2011.10.031.

10. Yadav, A. K., \& Bhattacharyya, S. (2014). Transient analysis of subcritical/supercritical carbon dioxide based natural circulation loops with end heat exchangers: Numerical studies. International Journal of Heat and Mass Transfer, 79, 24-33. https://doi.org/10.1016/j.ijheatmasstransfer.2014.07.068.

11. Doganay, S., \& Turgut, A. (2015). Enhanced effectiveness of nanofluid based natural circulation mini loop. Applied Thermal Engineering, 75, 669-676. https://doi.org/10.1016/j.applthermaleng.2014.10.083.

12. Cheng, H., Lei, H., \& Dai, C. (2017). Heat Transfer of a Single-Phase Natural Circulation Loop with Heating and Cooling Fluids. Energy Procedia, 142, 3926-3931. https://doi.org/10.1016/j.egypro.2017.12.298.

13. Cheng, H., Lei, H., Zeng, L., \& Dai, C. (2019). Theoretical and experimental studies of heat transfer characteristics of a single-phase natural circulation mini-loop with end heat exchangers. International Journal of Heat and Mass Transfer, 128, 208-216. https://doi.org/10.1016/j.ijheatmasstransfer.2018.08.136.

14. Vijayan, P. K., Sharma, M., \& Saha, D. (2007). Steady state and stability characteristics of single-phase natural circulation in a rectangular loop with different heater and cooler orientations. Experimental Thermal and Fluid Science, 31(8), 925-945. https://doi.org/10.1016/j.expthermflusci.2006.10.003.

15. Kudariyawar, J. Y., Vaidya, A. M., Maheshwari, N. K., \& Satyamurthy, P. (2016). Computational study of instabilities in a rectangular natural circulation loop using 3D CFD simulation. International Journal of Thermal Sciences, 101, $193-206$. https://doi.org/10.1016/j.ijthermalsci.2015.11.003.

16. Krishnani, M., \& Basu, D. N. (2017). Computational stability appraisal of rectangular natural circulation loop: Effect of loop inclination. Annals of Nuclear Energy, 107, 17-30. https://doi.org/10.1016/j.anucene.2017.04.012.

17. Zvirin, Y., Jeuck, P. R., Sullivan, C. W., \& Duffey, R. B. (1981). Experimental and Analytical Investigation of a Natural Circulation System with Parallel Loops. Journal of Heat Transfer, 103(4), 645. https://doi.org/10.1115/1.3244521. 
18. Nayak, A. K., Gartia, M. R., \& Vijayan, P. K. (2008). An experimental investigation of single-phase natural circulation behavior in a rectangular loop with Al2O3 nanofluids. Experimental Thermal and Fluid Science, 33(1), 184-189. https://doi.org/10.1016/j.expthermflusci.2008.07.017.

19. Forster, Fred \& Bardell, Ron \& A. Afromowitz, $M \&$ R. Sharma, $N \&$ Blanchard, A. (1995). Design, fabrication and testing of fixed-valve micro-pumps. Proceedings of the Fluids Engineering Division of the ASME - FED. 234.

20. Gamboa, A. R., Morris, C. J., \& Forster, F. K. (2005). Improvements in Fixed-Valve Micropump Performance Through Shape Optimization of Valves. Journal of Fluids Engineering, 127(2), 339. https://doi.org/10.1115/1.1891151.

21. Zhang, S., Winoto, S. H., \& Low, H. T. (2007). Performance Simulations of Tesla Microfluidic Valves. 15-19. https://doi.org/10.1115/mnc2007-21107.

22. Thompson, S. M., Ma, H. B., \& Wilson, C. (2011). Investigation of a flat-plate oscillating heat pipe with Tesla-type check valves. Experimental Thermal and Fluid Science, 35(7), 1265-1273. https://doi.org/10.1016/j.expthermflusci.2011.04.014.

23. Nobakht, A. Y., Shahsavan, M., \& Paykani, A. (2013). Numerical study of diodicity mechanism in different tesla-type microvalves. Journal of Applied Research and Technology, 11(6), 876-885. https://doi.org/10.1016/S1665-6423(13)71594-3.

24. Thompson, S. M., Jamal, T., Paudel, B. J., \& Walters, D. K. (2014). Transitional and Turbulent Flow Modeling in a Tesla Valve. V07BT08A027. https://doi.org/10.1115/imece2013-65526.

25. Thompson, S. M., Paudel, B. J., Jamal, T., \& Walters, D. K. (2014). Numerical Investigation of Multistaged Tesla Valves. Journal of Fluids Engineering, 136(8), 081102. https://doi.org/10.1115/1.4026620.

26. De Vries, S. F., Florea, D., Homburg, F. G. A., \& Frijns, A. J. H. (2017). Design and operation of a Tesla-type valve for pulsating heat pipes. International Journal of Heat and Mass Transfer, 105, 1-11. https://doi.org/10.1016/j.ijheatmasstransfer.2016.09.062.

27. Rajat, A. C., (2019). Stability analysis of Tesla valve based natural circulation loop for decay heat removal in nuclear power plants. in Proc. IEEE 2019 Advances in Science and Engineering Technology International Conferences, https://doi.org/10.1109/ICASET.2019.8714209 
